University of Nebraska - Lincoln

DigitalCommons@University of Nebraska - Lincoln

7-2016

\title{
Contribution of Fiber Undulation to Mechanics of Three- Dimensional Collagen-I Gel
}

Shengmao Lin

University of Nebraska-Lincoln, linshengmao@gmail.com

Linxia Gu

University of Nebraska-Lincoln, gul@fit.edu

Follow this and additional works at: https://digitalcommons.unl.edu/mechengfacpub

Part of the Mechanics of Materials Commons, Nanoscience and Nanotechnology Commons, Other Engineering Science and Materials Commons, and the Other Mechanical Engineering Commons

Lin, Shengmao and Gu, Linxia, "Contribution of Fiber Undulation to Mechanics of Three-Dimensional Collagen-I Gel" (2016). Mechanical \& Materials Engineering Faculty Publications. 165.

https://digitalcommons.unl.edu/mechengfacpub/165

This Article is brought to you for free and open access by the Mechanical \& Materials Engineering, Department of at DigitalCommons@University of Nebraska - Lincoln. It has been accepted for inclusion in Mechanical \& Materials Engineering Faculty Publications by an authorized administrator of DigitalCommons@University of Nebraska Lincoln. 


\title{
Contribution of Fiber Undulation to Mechanics of Three-Dimensional Collagen-I Gel
}

\author{
Shengmao $\operatorname{Lin}^{1}$ and Linxia Gu$u^{1,2}$ \\ 1 Department of Mechanical and Materials Engineering, University of Nebraska-Lincoln, \\ Lincoln, NE 68588-0656, USA \\ 2 Nebraska Center For Materials and Nanoscience, Lincoln, NE 68588-0656, USA
}

Corresponding author — L. Gu, lgu@unl.edu

\begin{abstract}
The collagen-I gel is extensively used as a scaffold material in tissue engineering due to its ability to mimic the extracellular matrix (ECM). In this study, the mechanics of collagen-I gel is investigated using a numerical model of three-dimensional collagen network. The resulted mechanical behavior was validated against the published experimental data. Results illustrated that fiber alignment was dominated in the low strain region, and its transition to stretching dominated phenomena at higher strain led to the strain stiffening of collagen gel. The collagen undulation at the microscopic level was found to delay the initiation of strain stiffening.
\end{abstract}

\section{Introduction}

The ability to closely mimic the extracellular matrix (ECM) scaffolds is one of the most critical requirements in tissue engineering. ${ }^{[1]}$ Understanding the mechanism of how ECM transduces force and deformation from the macro-level (tissue-organ) to the micro-level (cell-fiber) is very important if we want to mimic its mechanical behavior. Collagen, being the main load-bearing component of ECM, significantly contributes to the biomechanical strength of the tissue. The collagen-I gel provides a simple structure that can be studied using multiphoton microscopy ${ }^{[2,3]}$ and serves as a scaffold for growing artificial tissue and a three-dimensional (3D) environment for studying cell motility and tumor invasion. Understanding its mechanical behavior can help us design better biomaterials to make the best of its characteristic.

The strain stiffening effect is the most prominent mechanical property of collagen-I gel. The network becomes stiffer as the strain increases. The cause of this strain stiffening is still not well understood. There are mainly two mechanisms which have been used to explain its origin: (i) the microstructural nonlinearities of individual filaments ${ }^{[4]}$; (ii) network effect: composed of nonaffine deformations of multiple filaments and transition from bending regime to stretching regime. ${ }^{[5]}$ Despite these two different explanations, the mechanism of the strain-stiffening effect of collagen-I gel still remains to be elucidated.

The thermal undulation of collagen fiber is commonly seen in native tissue and a recent experiment done by R. Rezakhaniha et al. ${ }^{[6]}$ has quantified the waviness of collagen fiber using confocal microscopy. However, only one study ${ }^{[7]}$ has investigated the effect of thermal undulation in biopolymer networks using a two-dimensional model and none of them has focused on collagen gel. To resolve this issue, a 3D undulated collagen network was built and conditions.

\section{Materials and Methods}

A 3D collagen network $(40 \mu \mathrm{m} \times 40 \mu \mathrm{m} \times 40 \mu \mathrm{m})$ was generated by adding lines from random points with random angles until reaching the desired volume fraction. The maximum and minimum length of the line is set to be $8 \mu \mathrm{m}$ and $4 \mu \mathrm{m}$ respectively, and the desired volume fraction is $0.073 \%$ which represents the $1 \mathrm{mg} / \mathrm{ml}$ 
collagen gel. The volume fraction is estimated by the product of the mass density of collagen in solution $\left(\rho_{c}=1 \mathrm{mg} / \mathrm{ml}\right)$ and the specific volume of collagen $\left(v_{c}=0.73 \mathrm{ml} / \mathrm{g}\right) \cdot{ }^{[8]}$ Each line is modeled by beam elements with length equal or smaller than $2 \mu \mathrm{m}$ which was proved to be the average length between crosslinks in collagen networks. [9] The diameter of each fiber is assumed to be $62 \mathrm{~nm}$ corresponding to the collagen gel polymerized at $37^{\circ} \mathrm{C}$ and $\mathrm{pH} 7.4 .^{[2]}$

To achieve the connectivity of the network, the crosslinks between lines were then generated when the distance between two nodes is less or equal than $800 \mathrm{~nm}$. The isolated beam elements were then removed to reduce computational cost since they won't take loading and contribute to the network stiffness.

Initially, a total of 1934 collagen fibers are generated as shown in Figure 1(a). After crosslinking and removing the isolated elements, 2360 crosslinks were generated and the number of collagen fiber is reduced to 1130 . Here we defined the crosslink density as the number of crosslinks per collagen fiber. In this case, the crosslink density is 2.09 .

The Young's modulus of collagen fiber is defined as 50MPa which is also used in the study of Stein et al.'s ${ }^{[5]}$ and close to the value $79 \mathrm{MPa}$ chosen by Stylianopoulos et al. ${ }^{[10]}$ The crosslinks were assumed to have the same material property to collagen fibers. At the intersection points between crosslinks and collagen fibers, no sliding motion is allowed, which means all the displacements and rotations at the intersection point are fixed. Uniaxial tensile loading along the $x$-direction is applied and solved using nonlinear finite element analysis software ABAQUS 6.12 (Simulia, Providence, RI). The network strain is calculated by dividing the displacement by the cubic edge length $(40 \mu \mathrm{m})$. The macroscopic stress is calculated by the total reaction force at the $y$ - $z$ plane divided by the cross-sectional area. The crosssectional area is estimated by the area of the single collagen fiber times the number of collagen fiber passing through that plane.

In addition, the effect of thermally induced undulation is also investigated. To quantify the thermally induced undulation of collagen fiber, the straightness parameter $\left(P_{\mathrm{s}}\right)$ is introduced:

$$
P_{s}=L_{0} / L_{f}
$$

where $L_{0}$ is the direct distance between two ends of collagen fiber, and $L_{f}$ is the actual length of the collagen fiber. In the study by Rezakhaniha et al., ${ }^{[6]}$ the straightness parameter of the collagen fiber is measured at the zero-stress state using the fluorescence microscope and distributed with a beta distribution (mean value 0.71 , variance 0.028 ). The mean values 0.71 and 0.83 are adopted to investigate the role of thermally induced undulation on collagen network mechanical performance.

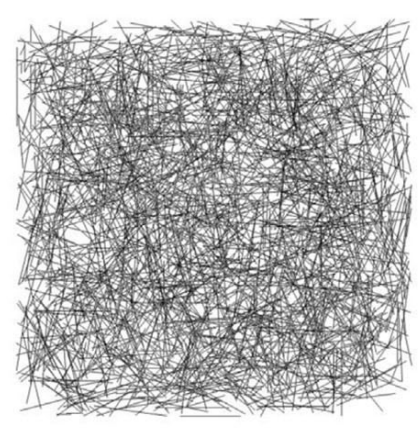

(a)

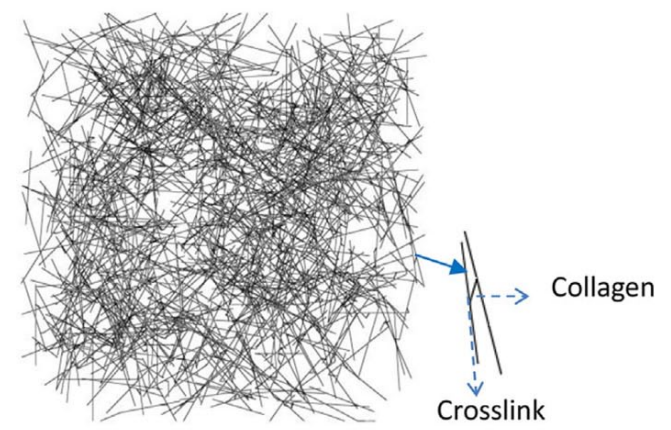

(b)

Figure 1. (a) Projection along z-axis of initial network; (b) Projection along z-axis after crosslinking and removing isolated elements. 

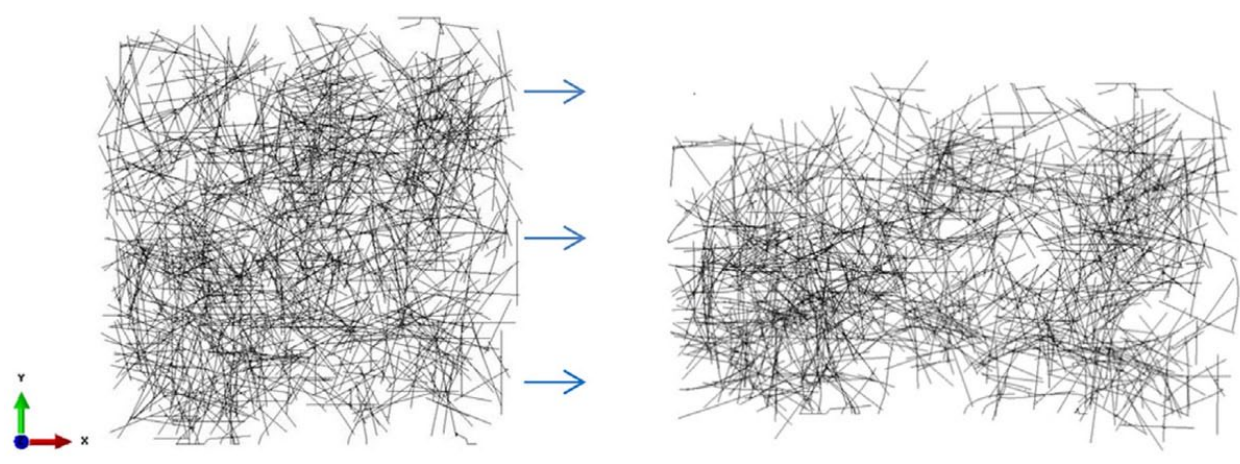

Figure 2. Collagen network at $0 \%$ strain (left) and $40 \%$ strain (right) in the $X-Y$ Plane.

\section{Results}

\section{Validation Case}

To test the accuracy of our modeling approach, the model specification is adjusted based on the experimental work of Roeder et al. ${ }^{[3]}$ In this experimental work, the uniaxial tensile test was performed on the collagen gels and the confocal microscopy was used to get the microstructural information about the collagen gels. Based on the data, the fiber diameter was adjusted to $435 \mathrm{~nm}$ and the crosslinks are formed when the distance between two nodes is less than $450 \mathrm{~nm}$. The Young's modulus of collagen is changed to $79 \mathrm{MPa}$. The collagen network is subjected to $40 \%$ extension in $\mathrm{x}$-direction as shown in Figure 2. The stress-strain relationship in the tensile direction is compared to the experimental results in Figure 3. A good agreement is observed between the experimental results and the simulation results which validates our numerical model.

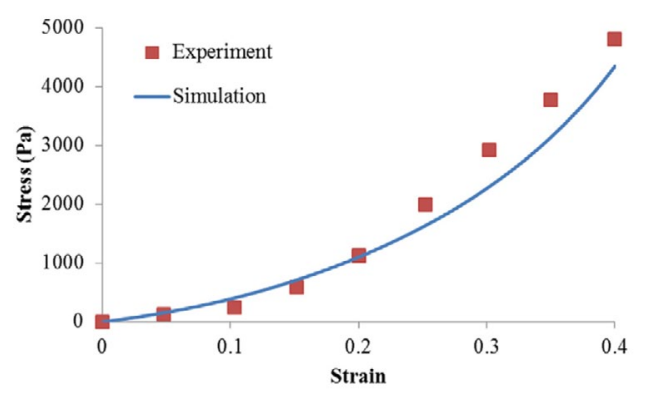

Figure 3. Stress-strain relationship for experimental (dots, (3)) and simulation results.

\section{Strain Stiffening Effect in the Baseline Model}

The strain stiffening effect is observed in the strain-stress relationship in Figure 3. To explore the mechanism behind, the possibility distribution of collagen orientation in degrees at every 0.05 strain are calculated. Figure $4(a, b)$ shows the possibility distribution at $0 \%$ and $40 \%$ strain in the $X-Y$ plane. An obvious increase possibility is observed at the 0 degree from the $0 \%$ strain to $40 \%$ strain. To quantify this change, the orientation possibility between the -15 to +15 degrees are summed in both $X-Y$ and $X-Z$ planes and plotted in Figure 4 (c). From this plot, the alignment of the collagen network is existing in the $X-Z$ plane throughout the whole stretching process. However, in the $X-Y$ plane, the alignment of the fiber network starts at $20 \%$ strain and ends at $30 \%$ strain. Therefore, the fiber alignment is strongest in the strain region from $20 \%$ to $30 \%$. Based on these observations, the stress-strain curve is divided into three regions: toe, transition and linear, as shown in Figure 4(e). The modulus of the network increases from 49.9 $\mathrm{Pa}$ at the toe region to $157.7 \mathrm{~Pa}$ at the linear region.

\section{Effect of Collagen Undulation}

The effect of collagen undulation is investigated by comparison between the straight and undulated collagen networks. Each collagen fiber has undulation shape with 0.71 and 0.83 straightness parameter, respectively shown in Figure 5(a) and (b). By stretching the network in the $x$-direction, the stress-strain curves for three different 


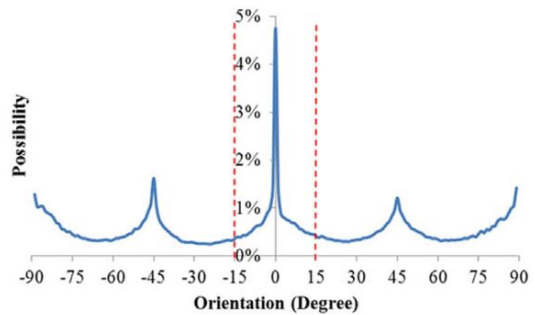

(a)

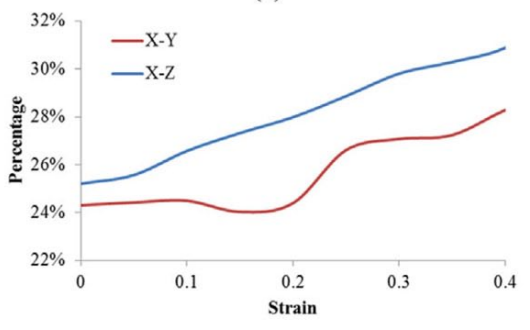

(c)

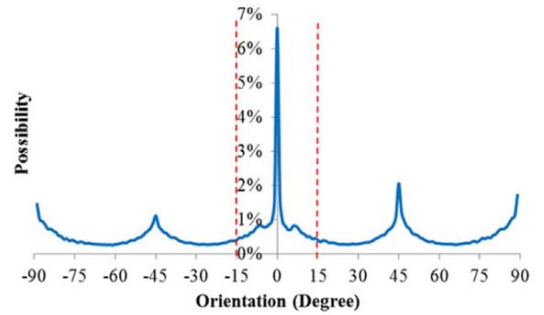

(b)

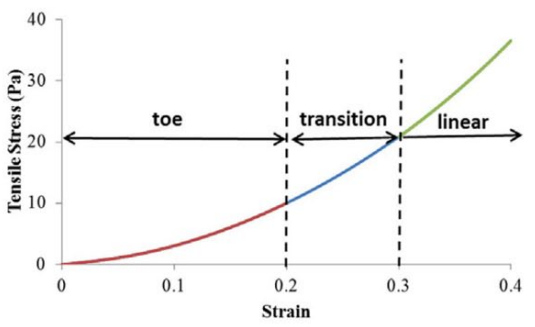

(d)

Figure 4. Collagen Orientation possibility in degrees at $0 \%$ strain (a) and $40 \%$ (b) in $X-Y$ plane; (c) summation of orientation possibility from -15 to +15 degrees at different strain in $X-Y$ and $X-Z$ planes; (d) stress-strain relationship dividing into three regions.

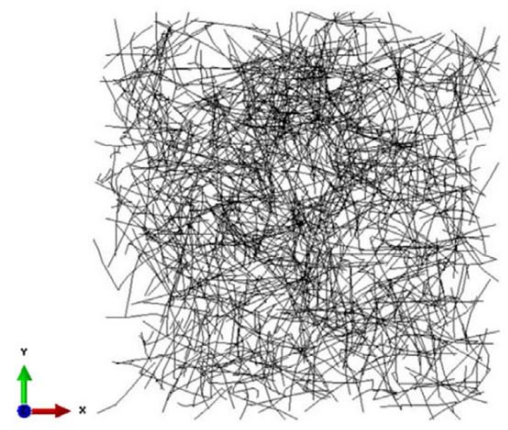

(a) $\mathrm{P}_{\mathrm{s}}=0.71$

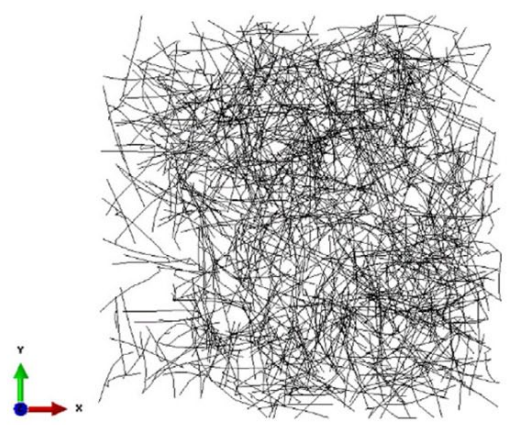

(b) $\mathrm{P}_{\mathrm{s}}=0.83$

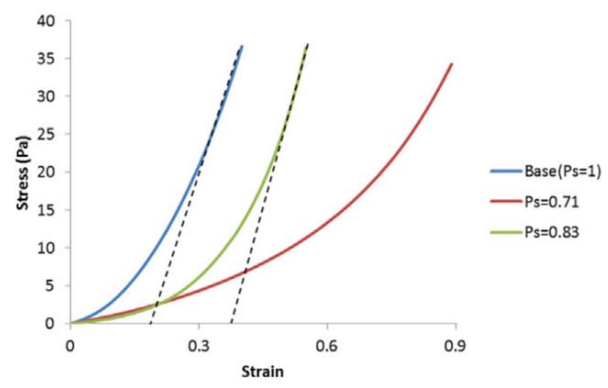

(c)

Figure 5. Undulated collagen network for (a) $P_{s}=0.71$ and (b) $P_{s}=0.83 ;(c)$ Stress-strain curve between straight and undulated collagen network. 
networks are plotted in Figure 5(c). A delay in strain stiffening is found when compared with the straight with the undulated collagen networks. In addition, an increase in delay of strain stiffening is found when the straightness parameter is decreased. However, there is not much difference in network linear modulus between the straight network (157.7 Pa) and the undulated network with 0.83 straightness parameter (177.7 Pa). The linear modulus of undulated network with 0.71 straightness parameter is not compared here since it does not reach the linear regime until the $90 \%$ strain.

\section{Discussion}

A three-dimensional collagen network accounting for the fiber-fiber interaction has been constructed to investigate the mechanism during the uniaxial stretching. Many previous constitutive models ${ }^{[11,12]}$ assume fibers act independently in the network while in many experimental studies, ${ }^{[13-15]}$ the crosslinks play an important role in determining its mechanical property which indicates the importance of fiber-fiber interaction. Considering the existence of the crosslink in our model allows us to account for the fiberfiber interaction. By adjusting model parameters according to the experiment, our model has been matched to the experimental work by Roeder et al. ${ }^{[3]}$ in terms of the stress-strain relationship in the stretching direction. By monitoring the change of fiber orientation angle during the stretching in both perpendicular planes, the strain stiffening effect is explained by the fiber alignment to the stretching direction during the deformation.

The role of collagen undulation is investigated by comparing the straight network with two undulated networks with different straightness parameters. The role of thermal undulations in biopolymer networks has been studied by Onck et al. ${ }^{[7]}$ using a two-dimensional network model which shows that filament reorientation is still the dominant mechanism while the thermal undulations only postpone the onset of stiffening. Using our model, we showed that the delaying in strain stiffening is still valid in threedimensional collagen networks and the effect of delay is increased when the straightness parameter is decreased.
The current study provides an improved understanding of the mechanics of collagen network using a computational approach. The modeling approach can be applied to other biopolymer networks or used as a base model to study the cell-ECM interactions. However, it is still limited that no breakdown or slippage is allowed at the crosslink which usually happens at the large strain. And it is designed for capturing the static behavior of collagen networks; the dynamics effect could be captured if the viscoelastic behavior of collagen ${ }^{[16]}$ is considered.

Conflict of Interest Statement - Neither of the authors has a conflict of interest.

Acknowledgments - This work was supported in part by the National Science Foundation CAREER award (CBET-1254095), and UNL Research Council Interdisciplinary Grant.

\section{References}

[1] D. E. Ingber, Mechanical and chemical determinants of tissue development. Principles of tissue engineering 2000, 101.

[2] C. B. Raub, V. Suresh, T. Krasieva, J. Lyubovitsky, J. D. Mih, A. J. Putnam, et al., Biophys. J. 2007, 92, 2212.

[3] B. A. Roeder, K. Kokini, J. E. Sturgis, J. P. Robinson, S. L. Voytik-Harbin, J. Biomech. Eng. 2002, 124, 214.

[4] C. Storm, J. J. Pastore, F. C. MacKintosh, T. C. Lubensky, P. A. Janmey, Nature 2005, 435, 191.

[5] A. M. Stein, D. A. Vader, D. A. Weitz, L. M. Sander, Complexity 2011, 16, 22.

[6] R. Rezakhaniha, A. Agianniotis, J. Schrauwen, A. Griffa, D. Sage, C. Bouten, et al., Biomech. Model. Mechanobiol. 2012, 11, 461.

[7] P. Onck, T. Koeman, T. Van Dillen, E. Van der Giessen, Phys. Rev. Lett. 2005, 95, 178102.

[8] D. J. Hulmes, A. Miller, Quasi-hexagonal molecular packing in collagen fibrils, 1979.

[9] S. B. Lindstr€om, D. A. Vader, A. Kulachenko, D. A. Weitz, Phys. Rev. E 2010, 82, 051905.

[10] T. Stylianopoulos, V. H. Barocas, Comput. Methods Appl. Mech. Eng. 2007, 196, 2981. 
[11] K. L.Billiar,M. S. Sacks, J. Biomech. Eng. 2000, $122,327$.

[12] N. J. Driessen, C. V. Bouten, F. P. Baaijens, J. Biomech. Eng. 2005, 127, 494.

[13] P. L. Chandran, V. H. Barocas, J. Biomech. Eng. 2004, 126, 152.

[14] D. Vader, A. Kabla, D. Weitz, L. Mahadevan, PLoS One 2009, 4, e5902.
[15] M.-T. Sheu, J.-C. Huang, G.-C. Yeh, H.-O. Ho, Biomaterials. 2001, 22, 1713.

[16] R. Puxkandl, I. Zizak, O. Paris, J. Keckes, W. Tesch, S. Bernstorff, et al., Philos. Trans. R. Soc. Lond. B Biol. Sci. 2002, 357, 191. 